Revue européenne des sciences sociales

European Journal of Social Sciences

XLIV-134 | 2006

Quel(s) défi(s) pour les sciences sociales à l'heure de la mondialisation?

\title{
Archéologie de l'imaginaire du feu, le principe de précaution des origines ou de la machine de Marly à la centrale nucléaire
}

\section{Alain Gras}

\section{OpenEdition}

\section{Journals}

Édition électronique

URL : http://journals.openedition.org/ress/291

DOI : $10.4000 /$ ress.291

ISSN : $1663-4446$

\section{Éditeur}

Librairie Droz

\section{Édition imprimée}

Date de publication : 1 juin 2006

Pagination : 129-137

ISBN : 9-782-600-01095-5

ISSN : 0048-8046

\section{Référence électronique}

Alain Gras, « Archéologie de l'imaginaire du feu, le principe de précaution des origines ou de la machine de Marly à la centrale nucléaire », Revue européenne des sciences sociales [En ligne], XLIV-134 | 2006, mis en ligne le 14 octobre 2009, consulté le 19 avril 2019. URL : http:// journals.openedition.org/ress/291; DOI : 10.4000/ress.291 


\section{ARCHÉOLOGIE DE L'IMAGINAIRE DU FEU, LE PRINCIPE DE PRÉCAUTION DES ORIGINES OU DE LA MACHINE DE MARLY À LA CENTRALE NUCLÉAIRE}

Evoquer l'image du feu c'est dès l'abord constater l'ambiguïté profonde avec laquelle nous appréhendons cet élément. Il est célébré comme une innovation décisive dans l'histoire de l'humanité et sa chaleur ne laisse pas d'être équivoque si l'on évoque sa flamme. Moyen de cuire les aliments, il représente une étape importante dans l'hominisation, mais son pouvoir est destructeur et réduit aussi la vie à néant.

Instrument de purification rituelle dans la plupart des religions, il est aussi le grand sacrificateur dans l'holocauste (de holos, tout, kaulein, brûler), terme qui aujourd'hui désigne spécifiquement le plus grand drame connu de l'humanité historique, une tragédie contemporaine de l'âge thermoindustriel.

On retrouve aussi là l'ambivalence première des origines: il est ambigu mais aussi ambivalent.

En effet, lorsque le feu vient du ciel, l'association avec le soleil se fait aisément, mais il sort aussi des profondeurs de la terre et les éruptions volcaniques confirment spectaculairement sa présence au-dessous de nous. C'est pourquoi les deux formes du feu se retrouvent stylisées dans l'espace indo-européen dans deux récits fondamentaux, celui de Prométhée dans l'antiquité grecque et celui de l'embrasement final du Ragnarök dans la mythologie scandinave et plus largement dans la tradition indo-européenne des quatre âges de l'humanité.

Lorsque l'homme l'apprivoisa, il n'est par conséquent sans doute pas totalement ridicule de penser que l'innovation «feu » suscita des interrogations dès le début, c'est-à-dire il y a quelques centaines de milliers d'années. Le roman plein d'humour du sociologue Roy Lewis ${ }^{1}$, Pourquoi j' ai mangé mon père, bâti autour de supposés débats sur l'adoption de cette innovation chez nos lointains ancêtres, nous raconte comment "l'industrieux Edouard voulant domestiquer le feu embrase toute la forêt». Je serais tenté de dire que cette situation vraisemblable il y a quatre cent mille ans peut aussi servir de métaphore pour la manière dont la machine à vapeur a transformé le monde. Les récits sur l'origine du feu gardent trace de cette ambiguïté première. On la découvre dans les récits collectés par le célèbre ethnologue anglais Frazer dans le tome Mythes sur l'origine du feu de son grand ouvrage Le rameau d'or ${ }^{2}$.

Lewis Roy, Pourquoi j’ai mangé mon père, Paris, Pocket, 2000.

Frazer J. G., Le rameau d' or, Paris, Laffont,1998, qu'utilise largement Gaston Bachelard dans son fameux ouvrage La psychanalyse du Feu, Paris, Gallimard, 1985. 
Aujourd'hui, le feu a gagné toute la planète. Imaginons, par exemple, qu'un extra-terrestre débarque sur la Terre avec la capacité de voir les résidus d'une combustion dans les objets à l'entour et que le rouge marque sa présence. L'univers quotidien de notre société deviendrait alors un paysage à tonalité pourpre semblable à un ciel de crépuscule l'été. Pratiquement toutes les autres couleurs seraient écrasées par cette teinte.

Un siècle et demi auparavant, qu'aurait-il vu dans les rues de nos villes ou dans les terres cultivées de nos campagnes? Une dominante couleur gris, celle de la pierre en ville, modulée par le rouge du ciment ou de la chaux, du zinc du toit aussi mais dans les rues la douce lueur du pavé du Mac Adam (trois couches de pierres concassées pour drainer le sol), le vert des vêtements portés aussi bien par les élégants que les classes pauvres, le vert de la prairie où poussaient le lin, le chanvre; mais aussi le cuir ou la soie, venue également de la vie animale - les vers sur le mûrier; le jaune des pays du soleil où naît le coton amené chez nous par le vent qui soufflait dans les voiles de grands bateaux en bois, le marron de ce bois des diligences, fiacres et autres carrosses dont seuls quelques bricoles de l'attelage, cerclages de roues, fer des sabots et châssis rougeoyaient. Dans le fond, le train annonçait le changement de couleur mais il n'était encore qu'un élément fugitif du paysage.

Trêve de métaphore, la conscience de la chaleur qui monte dans notre monde commence aujourd'hui à prendre forme dans la conscience de chacun, une forme souvent abstraite qui s'appelle $\mathrm{CO}_{2}$, trou d'ozone, gaz rare, ou plus généralement réchauffement climatique et effet de serre.

Non seulement toutes les techniques de la vie quotidienne tirent directement ou indirectement leur pouvoir d'un feu situé quelque part, là où il ne dérange pas (la centrale thermique ou l'usine nucléaire à la campagne par exemple) mais encore les objets que nous utilisons proviennent en grande partie de transformations obtenues par le feu: le métal, les résines synthétiques, les sacs, etc. La nourriture elle-même par les engrais, le transport, la réfrigération ou la congélation, la mise sous emballage qu'elle exige, consomme de la chaleur. Il est bien connu par exemple qu'un kilo de bœuf «vaut» 10 litres de pétrole pour sa production et son trajet jusqu'à l'assiette.

Comment en sommes-nous venus là? Si la question est idiote parce que ce qui est... devait être, alors il n'y rien à faire que d'observer la dynamique de ce lent recouvrement du monde par les scories du feu et d'écrire des essais sur la continuité de l'évolution technologique depuis la nuit des temps. Les tenants de ce discours admettent aisément, même si c'est depuis peu, les risques inévitables; mais ils comptent sur le même progrès technique pour les faire disparaître dans l'avenir. Cette façon de vouloir soigner le mal par le mal sans s'interroger sur les racines de ce mal se nomme politique de l'autruche et elle est idiote au vu de ce qui se passe sur la planète. A l'inverse, de toute logique, la question initiale ne l'est donc pas et il conviendrait par conséquent de s'essayer à une généalogie de l'omniprésence de cette chaleur en faisant l'hypothèse qu'il s'agit là d'une césure dans cette même évolution. Ceci veut dire qu'il faut chercher les racines de ce phénomène aussi bien dans la réalité matérielle que dans la manière dont il a été mis en place dans les esprits. Pour Nietzche, dont Michel Foucault a prolongé et actualisé la perspective à sa façon, les systèmes de valeurs s'inscrivent dans des 
luttes pour le pouvoir et l'apparition d'une nouvelle forme de penser n'est jamais fortuite de ce point de vue. Or l'objet technique est aussi une forme de pensée, il est une manière de faire exister le monde à l'entour. L'univers du quotidien et l'univers plus abstrait de la connaissance en tant que norme sont le résultat d'un ensemble de causes dont il serait absurde de faire la liste parce qu'elle sont indéfinissables précisément et sans doute innombrables. C'est donc bien plus la genèse de l'objet liée à celle de l'idée qui a du sens dans une interprétation du nouveau monde de la thermoindustrie. L'archéologie du précédent paragraphe nous a permis de découvrir un terreau pour faire germer les idées, il faut maintenant se rapprocher des racines de ce nouveau monde de la machine thermique.

Mais auparavant, un détour par la critique des fondements de l'évolutionnisme technologique est nécessaire. Ce détour, je le ferai du reste à plusieurs reprises car à chaque moment de l'exposé ressort l'idée centrale, apprise à l'école et sans cesse répétée, d'un développement progressif des techniques. Cette vision continuiste obscurcit la pensée parce qu'elle se donne comme évidente alors qu'elle n'est que naïveté construite.

L'apparition de la machine thermique comme «naturelle» s'appuie sur la croyance en une autonomie du développement technique, lequel suppose une continuité dans les changements de l'objet depuis son existence primitive jusqu'à aujourd'hui. En France Maurice Daumas en est le plus éminent représentant. En ouvrant un ouvrage qui parle des techniques à travers les âges, dans ce cadre théorique, nous nous heurtons sans cesse à l'évidence du progrès humain présenté à partir d'objets contemporains dont on reconstruit le parcours dans le temps de manière totalement abstraite, hors contexte. Ces objets racontent dans leur mode d'existence la fin provisoire d'une histoire où l'homme s'est montré un génial bricoleur avant que la raison n'émerge et en fasse un savant ou un ingénieur.

\section{DE LA BIOLOGIE À L'ANTHROPOLOGIE : LES ÉQUILIBRES PONCTUÉS ET LA CONSTRUCTION SOCIALE DE LA TECHNOLOGIE}

En général, les manuels nous apprennent qu'il est facile de suivre un objet à la trace et de concevoir ainsi son évolution. La voiture, exemple facile que je ne me lasse pas d'utiliser, prolonge, dans cette perspective, le char à bancs ou le carrosse, qui lui-même procède de l'attelage lourd à quatre roues des Gaulois successeur de celui à deux roues des Hyksos envahisseurs de l'Egypte dans les années 1600 av. J.-C. Même le téléphone portable ou internet ou tout autre média moderne se présente comme le prolongement contemporain d'une nécessité technique connue autrefois sous la forme de signaux de fumée ou de pigeons voyageurs!

Cette vision à rebours contient tout autant de vérité que celle qui nous fait remonter à nos ancêtres éloignés. Quelle est en effet le lien entre M. Tartanpion de l'an 2005 et celui que l'on trouve en remontant dans le temps il y a mille ans après un labyrinthe formé par des bifurcations successives. Une anecdote de Boris Cyrulnik vaut le peine d'être citée à ce propos. Répondant à un Monsieur «du Truc de la Chose du Machin descendant de Saint-Louis », il se présenta lui aussi de cette manière «Boris Gros Cro descendant de Cro Magnon »! Bel exemple de l'insignifiance de la quête du sens présent par le cheminement à rebours. 
L'ancêtre est toujours mythique et pas plus Saint-Louis que l'Homo Sapiens commun n'aident à comprendre la situation du sujet contemporain. Et il en va de même pour notre automobile: elle a quatre roues comme le char à bancs mais Saint-Louis et Cro Magnon avaient aussi deux jambes, est-ce que cela donne du sens pour comprendre les phénomènes dans leur réalité intime?

A côté de cet évolutionnisme radical qui est aussi un progressisme intégriste que l'on rencontre partout dans l'univers médiatique (comme en témoigne malheureusement l'Odyssée de l'espèce, téléfilm de Yves Coppens), une autre interprétation de la dynamique techno-logique présente bien plus d'intérêt. Les théories modernes de l'évolution, dont un des représentants les plus critiques et les plus iconoclastes n'est autre que le fameux Stephen J. Gould, célèbre pour son essai Quand les poules auront des dents ${ }^{3}$, insistent aussi sur cette incertitude du temps, en particulier dans la théorie dite des «équilibres ponctués», ou ponctuated equilibria. Cette théorie repose sur la constatation du fait que les tendances observées dans le monde fossile combine des périodes de stagnation dans l'évolution des espèces avec d'autres périodes de brusques accélérations. Elle fait donc l'hypothèse que, durant des périodes de temps plus ou moins longues, une espèce peut donner l'impression de disparaître alors qu'elle se protège dans un milieu rare en restant isolée dans une niche de l'écosystème. A la faveur d'un changement dans l'environnement, climatique, géologique ou autre, elle peut se retrouver plus adaptée que les concurrentes et montrer un dynamisme évolutif d'autant plus fort que l'espèce s'est endurcie dans la phase de récession. On peut interpréter ainsi le renouveau qui a suivi l'extinction quasi-totale des espèces au Permien il y a 250 millions d'années et la disparition plus connue des Dinosaures il y a 60 millions d'années.

L'histoire des êtres vivants repose donc sur une série de discontinuités (ponctuation) et non sur des mutations continues dues à une pression sélective constante. Je simplifie ce modèle de manière sans doute outrancière pour les puristes mais il m'importe d'en donner la substance anthropologique pour éclairer son usage dans un autre domaine que celui biologique. Cette théorie renouvelle le darwinisme en évacuant la notion de temps orienté dans l'évolution biologique puisque personne ne peut savoir quel sera demain le statut de ce qui est adapté aujourd'hui (par certains aspects elle le met en péril, comme certains créationnistes l'ont compris, mais je laisse la question ouverte dans un domaine qui n'est pas le mien). En outre, la notion de tendance dans ce cadre rejoint la forme évolutive que j'appelle trajectoire: l'évolution d'un être/objet se situe dans un temps discontinu et l' «évolution » orientée est ponctuée mais aussi ponctuelle, c'est-àdire limitée dans le temps avec une origine et une fin. Elle ouvre aussi vers une théorie positive du rôle des catastrophes dans l'histoire.

Avec ce modèle, par conséquent, la prévision sur la longue durée devient impossible. Durant une période, dont la durée peut varier grandement si on la mesure en années solaires, une espèce semble l'emporter, puis brusquement une

3 Gould St. J., Quand les poules auront des dents, Paris, Seuil, 1991; et Gould St. J., La vie est belle: les surprises de l'évolution, Paris, Seuil, 2004. 
autre, dont l'existence était cachée, remonte à la surface. Et plus tard, le jeu peut encore changer de donne, et ainsi de suite. Il n'y a pas de gagnant, sinon provisoire, au grand jeu de la vie.

L'exemple pourrait être suivi pour la technologie. Reprenons le cas le plus simple de l'automobile. Lorsque le pétrole était abondant et bon marché, le moteur à essence (c'est-à-dire à gaz liquide) était bien supérieur à celui à huile lourde d'hydrocarbure, le diesel. Mais si le prix du baril devient dissuasif, le pétrole moins raffiné, l'huile, retrouve son intérêt et si l' « huile de roche» vient à manquer alors le diesel l'emportera sur tous ses concurrents grâce à l'huile végétale, de colza, maïs, betterave, tournesol et autres. Le moteur à essence n'aura ouvert qu'une parenthèse dans l'histoire des techniques! Et ensuite... nul ne sait qui sera le prochain gagnant de la loterie. L'éveil de la puissance du feu endormi sous la terre et engrangé dans des roches (dont l'uranium) a bouleversé l'échiquier planétaire, mais est-ce là l'aboutissement d'un long processus de maturation comme on veut nous le faire croire, ou bien une longue parenthèse dans laquelle nous sommes enfermés?

Cette théorie des équilibres ponctués prend aussi une autre allure dans le domaine technique. Elle débouche - intellectuellement car il n'y aucune filiation directe - sur une autre théorie critique qu'il est convenu d'appeler «la construction sociale de la technologie» largement développée dans le monde anglo-saxon et défendue en France par Bruno Latour, sur un mode qui lui est propre et largement appliqué à la science. En parallèle avec la thèse biologique, ce modèle accorde une importance très grande au milieu mais cette fois ce milieu est humain, la niche est sociale. Je ne développerai pas ici les idées de ce courant mais je voulais signaler son importance parce qu'il aboutit par d'autres voies au même constat que celui de Gould et Evered: la bifurcation évolutive mêle au moment du choix de la voie d'avenir des êtres humains avec leurs désirs, leurs intérêts, leurs pulsions, et des artefacts avec leur déterminisme propre, lequel n'est en aucun cas décisif. Latour parle avec un peu d'emphase du «parlement des choses », je me contenterai de dire que l'évolution technologique (à ne pas confondre avec la croissance) procède par rupture de sens.

Et que l'on me permette une petite digression pour préciser ma pensée. La représentation conventionnelle en Occident du temps et de l'Etre, ou des êtres, des objets et pas seulement des humains, se greffe sur un modèle qui est aussi simpliste que stérile pour penser ce temps de manière créatrice. Prenons un cas hors du monde occidental. Les hindouistes pensent le monde en termes de cycles, un grand et des petits. Ainsi un grand cycle Manvantara se compose de cycles plus petits, les Mahayuga eux-mêmes constitués d' «âges » ou Yuga (les quatre âges de l'humanité des Anciens). Certains spécialistes de l'ésotérisme oriental, tel René Guénon, attribuent à ces âges un nombre d'années solaires, d'autres s'y refusent mais peu importe ce Manvantara n'est encore qu'un cycle du Kalpa, lequel correspond à un souffle de Brahma ${ }^{4}$ !

4 Un grand cycle Mahayuga est composé des quatre périodes dites yugas. Les yugas sont de plus en plus courts (en raison du déclin spirituel de l'humanité), selon une proportion de 4, 3, 2, 1. Le Satyayuga («l'Age de la Vérité») est le plus long; les trois autres yugas sont le Tretayuga (Treta peut se traduire par «triade»), le Dvaparayuga (l'Age du doute, de l'incertitude), et enfin le Kaliyuga (l'Age des conflits), qui est le plus court. Chaque yuga comporte une aurore, un jour et un crépuscule. Un manvantara comprend 71 Mahayuga et un kalpa comprend 1000 Mahayuga! Le Kali-yuga hindou 
Il faut bien sûr voir dans cette mathématique poético-métaphysique une image de l'homme dans le temps qui nous replace face à notre petitesse infinie. Toutefois cette fresque grandiose débouche sur une interrogation: où allons-nous en ce monde? Accompagnée de sa réponse: nulle part car le monde se recrée chaque jour à partir de rien (grâce à Brahma), il existe toujours quelque part dans l'un ou l'autre des recoins de cet espace-temps une nouveauté absolue. Le monde, en tant que phénomène total, crée du sens par des inventions radicalement neuves et en rupture radicale avec ce qui précède. La vision hindouiste s'oppose ainsi à celle occidentale que les principes juridiques du Droit canon catholique ont si bien exprimée au Moyen-Age, avant d'être reprises par la science moderne, Post hoc ergo propter hoc - «Après donc à cause de cela » - et Ex nihilo nihil fit - «de rien rien ne se fait». En deux adages tout est dit! Je partirai donc de l'hypothèse inverse qu'a formulée Andreu Solé dans le titre même de son ouvrage: (nous sommes) «Créateurs de monde » .

La bifurcation vers un univers entièrement fondé sur le pouvoir de la chaleur n'est pas une banalité anthropologique qui serait le résultat d'une nécessité inscrite dès l'aube de l'humanité dans la «nature humaine». Elle demande non pas à être expliquée car on ne conçoit pas le radicalement neuf qu'est la création dans une série de causalité (post hoc...) mais on peut la comprendre à partir d'une situation dans la niche. Voir son émergence comme une coalescence, une cristallisation, une précipitation, une apparition, au sens religieux du terme, d'un phénomène porteur d'un sens jusque là inconnu.

\section{NORIA, MACHINE DE MARLY ET TURBINE. UNE BIFURCATION IMPOSSIBLE?}

Si l'on compare dans l'histoire des idées les relations qu'ont entretenues les hommes avec les divers éléments, on note immédiatement que la différence là aussi est très grande. Alors que la terre, l'air, l'eau ont très tôt fait l'objet de mesures et d'expérimentations accompagnées de connaissances rudimentaires de leurs propriétés, il en va tout autrement du feu. Le terme même d'agri-culture exprime une connaissance (une culture) de la manière dont on peut jouer avec la terre et sa fécondité, de même que les machines qui empruntent leur force à l'eau et au vent exigent bien une compétence à la fois théorique et pratique autant qu'un savoir-faire. La simplicité avec laquelle la noria élève grâce à ses godets l'eau jusqu'à 30 mètres à Hama en Syrie (le diamètre de la roue) en utilisant le courant du fleuve ou de la rivière, ou bien l'extraordinaire virtuosité des ingénieurs romains qui obtenaient sur leurs aqueducs une pente rectiligne de $1 \mathrm{~mm}$ par mètre sur des distances supérieures à cent kilomètres, sont des exemples d'usage pacifique de l'énergie. Ils démontrent une connaissance réelle, intime, des forces de la

correspond à l'Age de Fer des Anciens Grecs et à l'Age du Loup de la tradition germano-scandinave (in la Völuspa). Selon la tradition shivaïte le Kaliyuga aurait commencé en 3600 avant J.-C., et sa période finale (ou crépuscule) en 1939. Voir pour une exégèse récente Tisserant J.-M., La guirlande de Kâli, Paris, Tredaniel, 1998.

5 Solé A., Créateurs de monde: Nos possibles, nos impossibles, Monaco, Ed. du Rocher, 2000. 
nature pour les récupérer sans les nier dans leur essence. Les temps modernes vont perdre cette sagesse et les architectes de Louis XIV seront incapables de terminer l'aqueduc qui devait détourner l'Eure pour les fastes de Versailles ${ }^{6}$. Cette période vit d'ailleurs surgir un délire de grandeur dans la maîtrise de l'eau qui n'est pas sans intérêt, car elle témoigne à la fois de la vanité et de la volonté de domination des forces naturelles et d'une créativité délirante dans la recherche de la captation de ces forces juste avant que ne se fassent jour, dans le même domaine, les premières tentatives fondées sur l'énergie fossile. Je veux parler de la machine de Marly qui alimentait les jets d'eau du Roi Soleil. Cette gigantesque pompe imaginée par un autodidacte de Liège, Renquin Sualem, et le Chevalier Arnold de Ville lui aussi liégeois, faisait remonter l'eau de la Seine de 165 mètres sur 1200 mètres jusqu'au point de départ de l'aqueduc qui la conduisait, après 634 mètres au réservoir de Louveciennes puis par un canal souterrain de 6 kilomètres vers d'autres réservoirs. Pour atteindre le haut de la colline de Marly trois relais puisards étaient nécessaires dont les pompes étaient actionnées par une partie de l'eau qui retombait, de même les quatorze grandes roues dont les aubes faisaient 12 mètres de diamètre étaient mues par le courant de la Seine et une chute artificielle. Un fabuleux système de bielles et de manivelles transformait la rotation en un mouvement alternatif complexe (voir figure ci-dessous) qui agissait sur les pompes. Au dire des voisins de l'époque dans les plaines à l'entour le bruit était épouvantable mais il signifiait aussi la grandeur du règne.

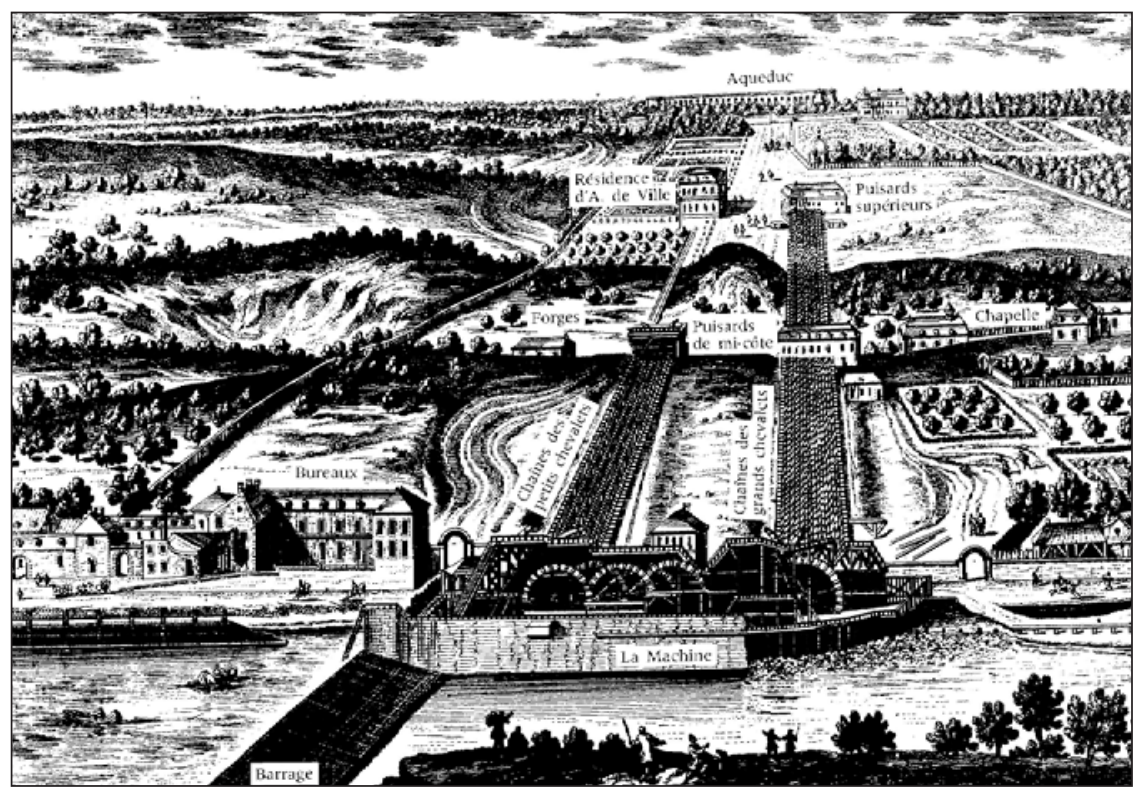

La Machine de Marly dans son environnement

${ }^{6}$ On peut en voir un morceau dans les Yvelines, près de Buc-sur-Yvette. 
On sait que les vannes des jets d'eau, situés à 37 mètres en contrebas du bout de l'aqueduc, étaient ouvertes à l'arrivée du Roi avec ses invités et refermées ensuite pour que l'eau puisse jaillir au fur et à mesure de la progression du cortège royal. Le rendement était donc très faible et il fallait sans doute un grain de folie et beaucoup de naïveté à Sualem pour avoir cru à ce projet. L'étanchéité des pompes laissait à désirer car l'alésage était loin d'être un travail de précision, le bois jouait, le fer aussi suivant la température, les peaux en cuir avaient du mal à résister à la pression et les innombrables engrenages étaient la cause de pannes récurrentes. Malgré tout, elle a réussi à fournir 3'000 $\mathrm{m}^{3}$ d'eau par jour à la cour (mais parfois deux fois moins en été prévu en théorie 6'000 $\mathrm{m}^{3}$ ) et elle a fonctionné tant bien que mal jusqu'en 1817, soit 133 ans. Ce qui n'est pas une mince durée pour un engin aussi compliqué.

Compliqué et pourtant très simple dans son objectif qui est la récupération de l'énergie naturelle de la gravitation pour lutter contre cette même gravitation. Admirable principe que la folie des grandeurs d'un roi mit en application dans un objet technique d'une ampleur inouïe pour l'époque. Malheureusement la notoriété de la machine de Marly ne suffit pas à mettre en place une réflexion sur les possibilités d'une telle technologie renouvelée par le savoir scientifique naissant. Elle n'ouvrit pas une nouvelle trajectoire et elle représente ainsi l'échec d'une pensée plus que l'échec d'une technique. A l'horizon, se profilait la machine de Newcomen et un siècle plus tard la pompe de Watt fermait cette bifurcation possible au profit de la puissance nouvelle tirée de l'énergie fossile. La machine de Marly dans son principe énergétique continue donc la noria (qui n'est pas une pompe) mais de manière monstrueuse et elle ferme la voie par son exagération au lieu d'ouvrir vers une autre bifurcation. On retrouvera tout de même, comme on le verra, l'idée de l'usage de la force hydraulique dans les moulins de la révolution industrielle au XVIII ${ }^{\mathrm{e}}$ et de la turbine au XIX ${ }^{\mathrm{e}}$ siècle, mais la pompe mue par l'énergie gravitationnelle sera abandonnée. Elle subsiste seulement dans la technique modeste du bélier. A l'approche du $\mathrm{XX}^{\mathrm{e}}$ siècle, la trajectoire des énergies renouvelables, dans ce domaine, sera fermée au moins jusqu'à nos jours, même si des éoliennes profiteront du vent pour pomper l'eau dans quelques lieux privilégiés d'où elles seront graduellement expulsées. Ainsi dans la plaine d'Ibiza, les pales inertes et les structures rouillées témoignent-elles tristement d'un passé d'autonomie des fermes maintenant branchées sur un réseau communal alimenté par une pompe centrale, évidemment thermique.

La machine de Marly monte à son paroxysme une volonté de puissance inscrite dans une voie technologique qui en réalité ne pouvait pas réaliser pleinement son objectif, parce que le seul recours aux énergies naturelles imposait une soumission à un ordre qui n'était pas politique. L'énergie fossile débloquera ce verrouillage mais un exemple récent ressemble à l'objet dont on parle. Le Concorde nous parlait le langage de la surmodernité, la vitesse, l'altitude, la splendeur des lignes, toutefois ses concepteurs n'avaient pas compris que le social ne pouvait se domestiquer, que la puissance de l'engin lui venait de l'extérieur et, par hasard ces années-là, il y eut la première grande crise de l'énergie, celle de l'OPEP.

La crise de 1973 fit basculer nos économies dans une nouvelle donne énergétique. Si l'on juge aujourd'hui, elle ne porta guère ses fruits sur le plan général car on oublia vite la nécessité de trouver d'autres voies mais pour le Concorde cet 
élément, ajouté à d'autres dont l'orientation commerciale vers le transport de masse à la place du transport élitiste, fut un facteur aggravant qui pesa lourdement dans la balance. La voie du supersonique civil fut ainsi abandonnée. A quelques années près, il en aurait peut-être été autrement. Concorde a-t-il échoué par hasard?

Je ne le crois pas car la politique de l'autruche du monde industriel, qui montre aujourd'hui ses vraies limites, ne pouvait donner qu'un sursis à ce genre de gouffre énergétique. Pourtant, si l'on ouvre le champ des bifurcations possibles, Concorde pouvait fort bien attendre son heure et survivre plus longtemps dans un système aéronautique fondé sur le transport de riches, et non plus de simples consommateurs (ce qui sera peut-être le cas de l'aviation civile demain). Mais il n'a pas pu trouver sa niche et l'accident de Gonesse l'a définitivement exclu du paysage social. Notons qu'ainsi à travers ces exemples, l'histoire réelle nous ouvre sans cesse des voies. Le temps humain est rythmé par le kä̈ros, ce moment créateur (le souffle de Brahma?) où tout peut basculer. On sait que les Grecs considéraient cette forme comme constitutive du temps avec le chronos, la forme de la continuité répétitive, et les éon, les très longues durées dans lesquelles se déploie le phénomène monde (l'équivalent des yuga). Dans ce modèle bien éloigné de la vision simplificatrice des temps modernes, la rupture ou bifurcation, en tant que phénomène essentiel de notre devenir est un garant de notre liberté. On peut ainsi juger qu'elle appartient aussi bien au phénomène technique. Plus précisément le temps de la technique fait partie intégrante du temps des hommes.

Bertrand Gille parlait de «société bloquée» en faisant allusion à la Chine, à l'empire musulman, aux Amérindiens. Mais après réflexion il ajoutait, outre les exemples anciens des Egyptiens et de la Grèce, les «primitifs », c'est-à-dire plus des trois quarts du monde à l'époque de la révolution industrielle ${ }^{7}$ ! Ne peut-on prendre le problème à l'envers car, en le posant à la manière de Gille, nous aboutissons à la conclusion qu'il y a un devoir être du progrès, une nécessité intrinsèque, ce qui ferait de cette histoire des techniques une philosophie déterministe de l'histoire, ce que refuse pourtant l'auteur. Prendre le problème à l'envers c'est donc s'interroger sur la manière dont les « occidentaux » ont rompu l'ancien pacte avec la nature. Et pour percer cette énigme, il me semble qu'il faut commencer par percer le mystère de la domination absolue qu'un seul des quatre éléments va exercer sur le fait technique. C'est à ce prix là que nous pourrons sortir de l'impasse dans laquelle s'est engagée la civilisation thermo-industrielle dont l'obsession de puissance sur la nature passe par l'usage immodéré du feu ${ }^{8}$. L'incendie s'étend, comment l'éteindre? Retournons visiter la sagesse des autres peuples... bloqués!

Centre d'Etude des Techniques, des Connaissances et de Pratiques (CETCOPRA) Université de Paris 1 Sorbonne gras@asterix.univ-paris1.fr

7 Gille B., Histoire des techniques, Paris, Gallimard/La Pléiade, chap. 6, p. 441 sq.

$\therefore$ Gras A., Fragilité de la puissance. Se libérer de l'emprise technologique, Paris, Fayard, 2003. 